\title{
Suppression of Roll Oscillation in Turning of Quadruped Robot by Asymmetric Amplification of Central Pattern Generator Output Waveform
}

\author{
Makoto Kitani ${ }^{1}$, Ryo Asami ${ }^{1}$, Noritaka Sato ${ }^{1, *}$, Yoshifumi Morita ${ }^{1}$, Tomofumi Fujiwara ${ }^{2}$, \\ Takahiro Endo 2 , Fumitoshi Matsuno ${ }^{2}$ \\ ${ }^{1}$ Morita and Sato Laboratory, Electrical and Mechanical Engineering, Nagoya Institute of Technology, Gokiso-cho, Showa-ku, Nagoya, Aichi 466-8555, Japan \\ ${ }_{2}^{2}$ Matsuno Laboratory, Kyoto University, Kyoto daigaku-Katsura, Nishikyo-ku, Kyoto 615-8530 Japan
}

\section{ARTICLE INFO}

\section{Article History}

Received 03 November 2018

Accepted 20 November 2018

Keywords

$\mathrm{CPG}$

quadruped robot

suppression of roll oscillation

\begin{abstract}
Quadruped robots experience excessive vibrations in the roll direction when turning by controlling their hip yaw joint. In the case of image-based teleoperation of the robot, the operator cannot aware the situation of the robot, due to excessive vibrations of the viewpoint of the image from the camera mounted on the robot. Especially, it is known that vibration in the roll direction is most likely to cause motion sickness. To overcome this problem, we proposed the asymmetric amplification of the output waveforms of central pattern generators. We implemented the proposed method on a robot in a dynamic simulator and verified the effectiveness of the proposed method, during the turning operation. As a result, we confirmed that the proposal method can suppress $43.7 \%$ vibration of the robot body in the roll direction and $7.4 \%$ vibration in the pitch direction compared with the conventional method.
\end{abstract}

(C) 2019 The Authors. Published by Atlantis Press SARL. This is an open access article distributed under the CC BY-NC 4.0 license (http://creativecommons.org/licenses/by-nc/4.0/).

\section{INTRODUCTION}

Legged robots can move on various terrain because they have legs and utilize their several degrees of freedom. They are controlled by planning their motion based on a strict model of a robot and an environment. However, planning their motion based on a strict model for various environments is quite complex, and inefficient to move various terrains at high speed.

To overcome this problem, Central Pattern Generators (CPGs) can be used. The mechanism of the CPG is related to rhythmical movements such as walking and swimming [1,2]. The CPG, an animal motion generation method, enables the motion of legged robots on various terrain using only one algorithm because of a pull-in phenomenon.

Because of the above advantages, CPGs have been used in robots in several studies. Kimura et al. implemented the CPG on a real robot. The robot "Patrush" can walk [3] and "Tekken4" can turn [4] on rough terrain. The turning motion is realized by controlling the hip yaw joint. However, the vibrations of the robot body are large because falling phenomenon in the roll direction occurred.

In the case of teleoperation of a legged robot, an operator controls a robot while watching the images from a camera mounted on the robot. Therefore, the image vibration should be small for the operator to understand the surroundings of the robot. In the experiment conducted by Kimura et al. [4], the robot experiences vibration in

"Corresponding author. Email: sato.noritaka@nitech.ac.jp the roll direction. Figure $1^{\text {a }}$ shows the graph of the posture of the main body (the roll and pitch direction) when turning on a flat ground [4]. According to the body roll angle (thick black line) in the graph, the robot vibrates, and it is finally overturned.

In the method proposed by Kimura et al., the camera image will vibrate so that teleoperation will be difficult. According to the study conducted by Tsubaki et al. [5], if the frequency of the image vibration is the same, a larger amplitude makes the operator uncomfortable. Therefore, in teleoperation, the image vibration should be small. Figure $2^{\mathrm{b}}$ illustrates a graph showing the relation between the subjective evaluation and the rotation angle for motion sickness [6]. Vibration in the roll direction is most likely to cause motion sickness at any speed of rotation.

The vibration of the robot body causes discomfort to the operator. There are many researches of robots installed CPG [7-9] without considering suppression of roll direction vibration. On the other hand, we found a research about suppression of quadruped robot body vibration [10]. However, the research did not consider of vibration during turning. Therefore, the purpose of this study is to suppress roll vibration in a legged robot during turning. To suppress roll vibration, we proposed a turning method using the asymmetric

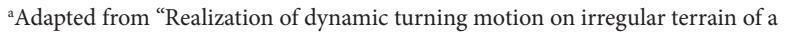
quadruped robot "TEKKEN4" with neural oscillators" by Fukuoka and Kimura [4], Copyright 2006 by The Japan Society of Mechanical Engineers. This image is remade for this paper.

bAdapted from "Visually induced motion sickness" by Ujike [6], Copyright 2007 by The Institute of Image Information and Television Engineers. 


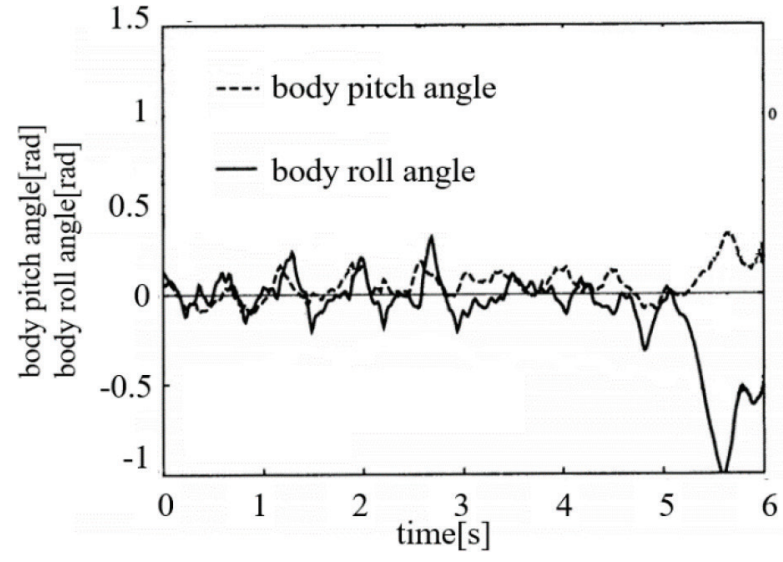

Figure 1 Tekken body angle in turning on flat land [4a].

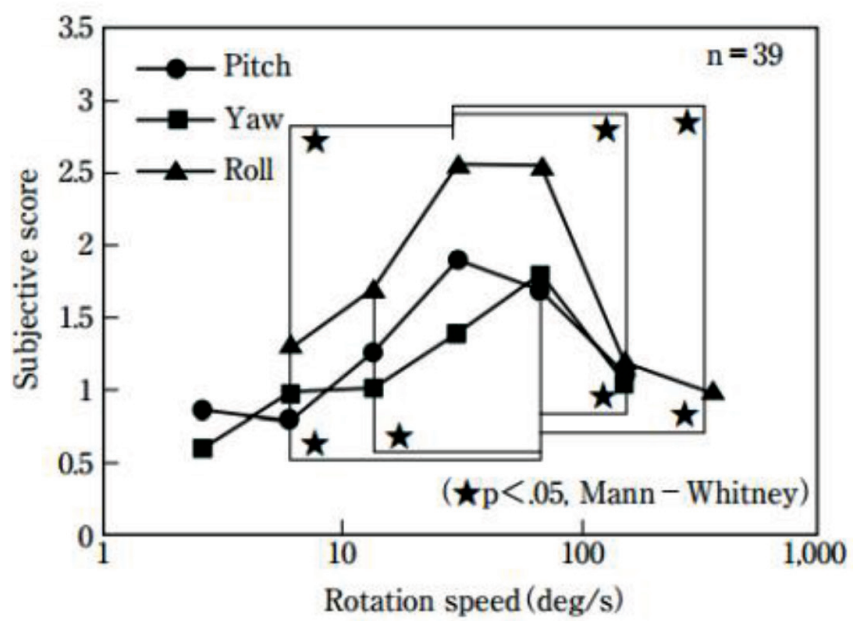

Figure 2 Subjective evaluation value of motion sickness with respect to speed of rotation when rotation relative to yaw, pitch, and roll axis is given in video $\left[6^{b}\right]$.

amplification of the CPG output waveform. In this study, we verified the effectiveness of the proposed method comparing with the previous method [4], that uses the yaw hip joint for turning, by carrying out an experiment using a dynamic simulator.

\section{WALKING ON FLAT GROUND USING CPG}

\subsection{Nonlinear First-order Simultaneous Differential Equations Constituting CPG}

The basic formula of the CPG is a nonlinear first-order simultaneous differential equation shown in Equations (1)-(3) [3].

$$
\begin{aligned}
\tau \dot{u}_{i} & =-u_{i}-\beta v_{i}+\sum_{j=1}^{n} w_{i j} y_{j}+u_{0}+\text { Feed }_{i} \\
\tau^{\prime} \dot{v}_{i} & =-v_{i}+y_{i} \\
y_{i} & =\max \left(0, u_{i}\right)
\end{aligned}
$$

$u_{i}, v_{i}$, and $y_{i}$ are the value, fatigue state, and output of the $i$-th neuron, respectively, $u_{0}$ is the steady input that generates oscillation, Feed ${ }_{i}$ is the feedback such as the joint angle, $\beta$ is the fatigue state factor, $\tau$ and $\tau^{\prime}$ are the time constants of $u_{i}$ and $v_{i}$, respectively, and $w_{i j}$ is the connection factor that determines the influence of the other neurons. A pair of CPGs was mounted on each joint, and four equations were formed corresponding to each leg. This method is based on the biped walking program [11] of the CPG where the connection factor is defined as follows:

$w_{1}$ - Between the extensor and flexor of the same joint.

$w_{2}$ - Between the right and left of the same extensor and flexor.

$w_{3}$ - Between the knee and hip joints of the extensor.

$w_{4}$ - Between the knee and hip joints of the flexor.

$w_{5}$ - Others.

Figure 3 shows the proposed CPG configuration. We generated a waveform for walking by multiplying the different amplification gain by the output value of the CPGs of each joint. The walking waveform is given by Equation (4).

$$
\theta_{\text {joint }}=K_{\text {joint }}\left(y_{E}-y_{F}\right)
$$

$\theta_{\text {joint }}$ is the target angle of the joint, $K_{\text {joint }}$ is the amplification gain at the joint, $y_{E}$ and $y_{F}$ are the CPG output values of the extensor and flexor, respectively.

\subsection{Robot for Experiment}

A robot, which is used in the experiment, is shown in Figure 4. We developed the robot using an open-source dynamic simulator, named "Gazebo". The CPG parameters are listed in Table 1.

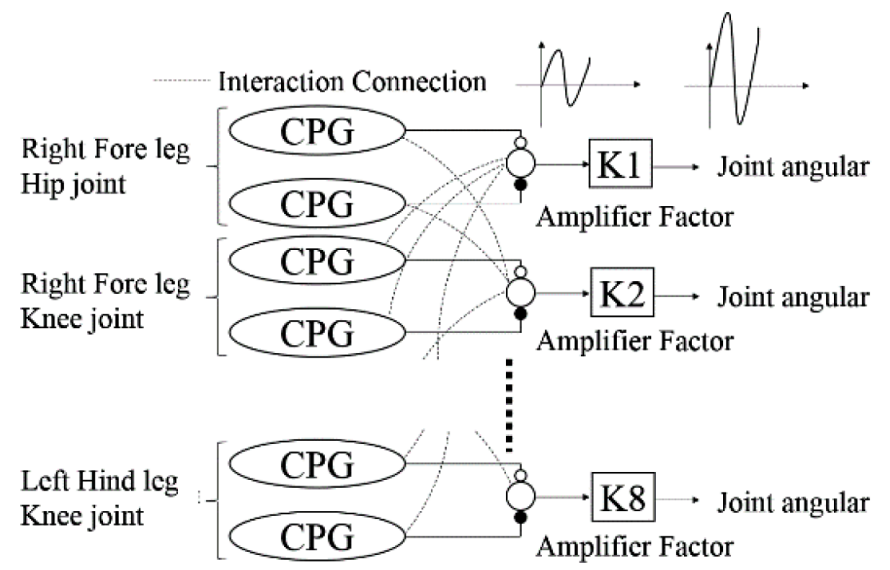

Figure 3 Proposed CPG configuration.

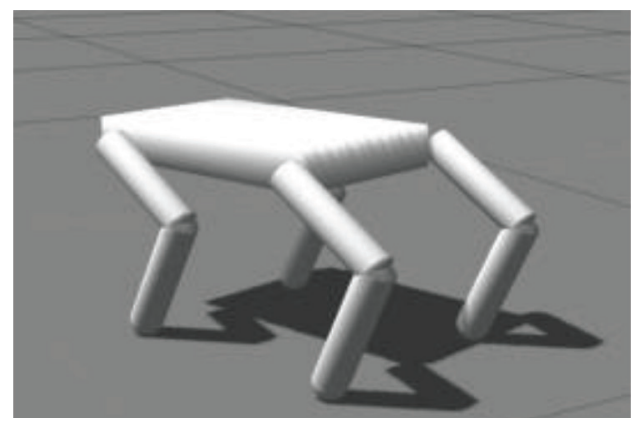

Figure $4 \mid$ A robot in simulator. 
Table $1 \mid$ CPG parameters

\begin{tabular}{llcl}
\hline Parameter & Value & Parameter & Value \\
\hline$u_{0}$ & 3.0 & $w_{1}$ & -2.0 \\
$\beta$ & 2.5 & $w_{2}$ & -1.0 \\
$\tau$ & 0.10 & $w_{3}$ & -0.50 \\
$\tau^{\prime}$ & 0.080 & $w_{4}$ & -0.30 \\
& & $w_{5}$ & 0 \\
\hline
\end{tabular}

Table 2 Amplification factor

\begin{tabular}{lcclcc}
\hline \multirow{2}{*}{ Joint name } & \multicolumn{2}{c}{ Gain (straight) } & & \multicolumn{2}{c}{ Gain (turning) } \\
\cline { 2 - 3 } \cline { 6 - 6 } & Right & Left & & Right & Left \\
\hline Hip & 2.2 & 2.2 & & 4.2 & 1.2 \\
Knee & 0.8 & 0.8 & & 1.8 & 0.8 \\
\hline
\end{tabular}

\section{TURNING MOVEMENT}

\subsection{Turning Method with Yaw Joint (Conventional Method)}

In the conventional method, the degree of freedom in the yaw direction is added to the hip joint. The turning radius can be changed by the angle of the yaw axis of the leg. However, in this method, the left front leg protrudes outside the robot. This increases the moment around the falling axis. We assumed that large vibrations would occur because of the increase in the moment.

\subsection{Turning Method without Yaw Joint (Proposed Method)}

To reduce the moment around the falling axis, we suggest a change in the amplification factor of the CPG output between the left and right legs. The robot is able to turn when the amplification factor of the joint angle on the outside of the turning circle is increased. The turning radius can be changed by changing the amplification factor. Table 2 lists the amplification factors at straight and turning, which is used in the experiment.

\section{VERIFICATION EXPERIMENT}

\subsection{Verification Condition}

To verify the proposed method, we used the Gazebo simulator as described in Section 2. We made the robot, which can walk on a flat ground using the simulator, installed two methods of turning, and evaluated their performances in the following sequence: adjusting the steady input to have the same speed and turning radius, measuring the roll angle of the robot body when the robot walks on the turning trajectory, and comparing the data. The following conditions were applied so that the robot can walk on the same turning radius.

- Conventional method:

Rotation angle of front leg - 0.15 [rad].

Stationary input - 2.7.
- Proposed method:

The amplification factor of the joint angle - The "gain (turning)" column of Table 2.

Stationary input - 3.0.

\subsection{Results and Discussion}

Figures 5 and 6 show the graph of the robot posture when turning in orbit shown as Figure 7. The peak-to-peak value of the roll angle in the conventional method is -0.2 to 0.15 [rad] and that in the proposed method is -0.1 to 0.1 [ rad].

In this study, we compared the conventional and proposed methods. As shown in Figure 5, the vibration in the roll direction could be suppressed. By comparing the maximum peak-to-peak value of both methods, we observed that the proposed method is able to suppress $43.7 \%$ vibration in the roll direction. Moreover, the proposed method suppressed $7.4 \%$ vibration in the pitch direction compared with the conventional method. We consider that the reason is the suppression of vibration around the falling axis. Moreover, motion sickness can be reduced because the vibration in the roll direction can be suppressed.

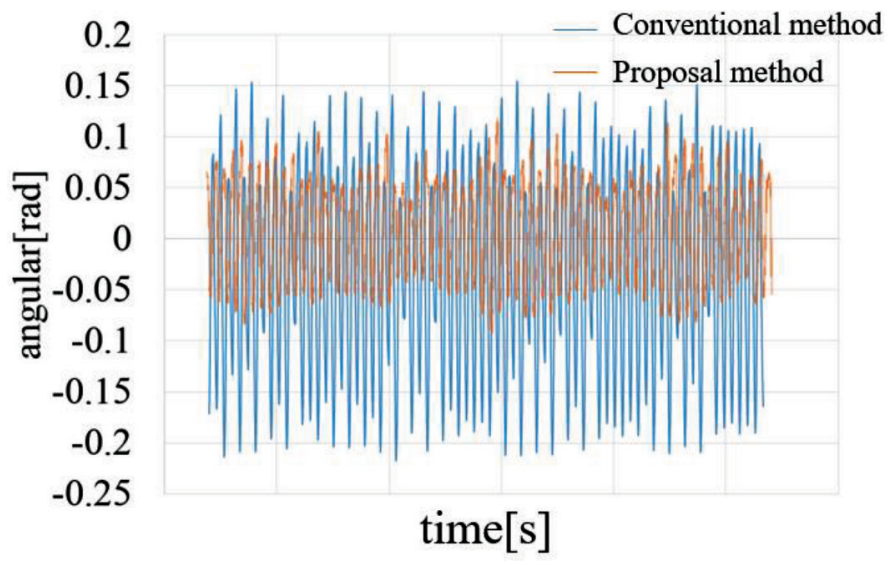

Figure 5 Robot body roll angle.

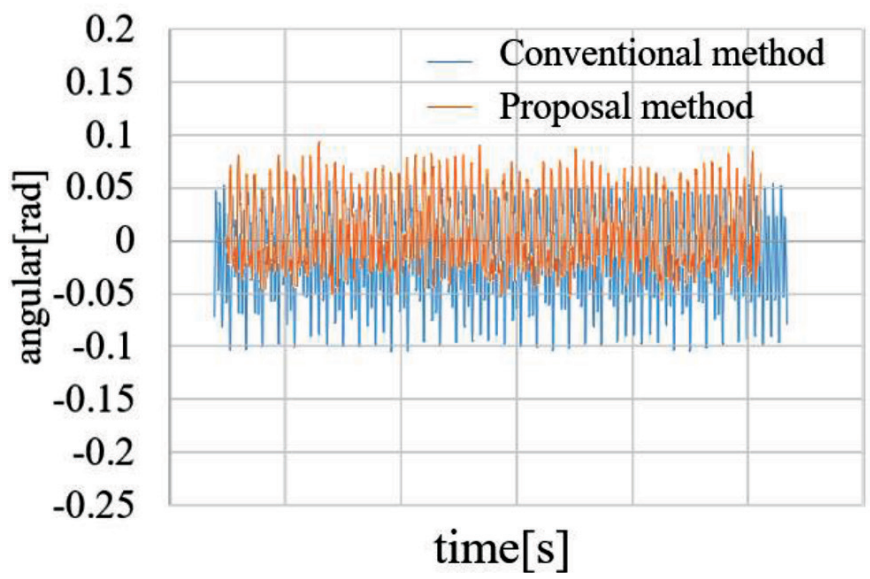

Figure 6 Robot body pitch angle. 


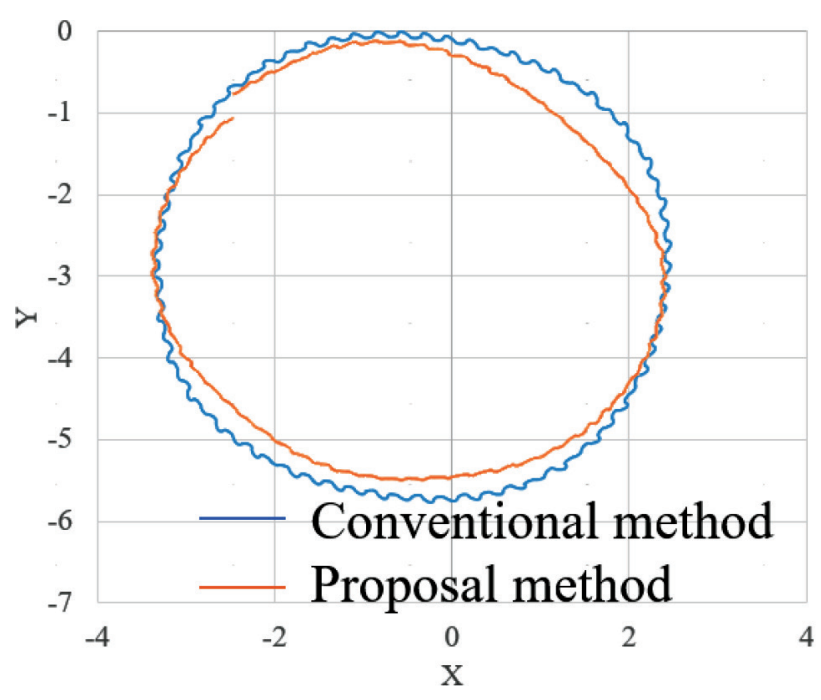

Figure 7 Orbit of robot turning.

\section{CONCLUSIONS AND FUTURE WORK}

The purpose of this study is to suppress roll vibration in the turning motion of a legged robot. We suggest a turning method that changes the amplification factor of the joint angle between the left and right legs to suppress the robot body vibration. We verified the effectiveness of the proposed method using a dynamic simulator compared with the conventional method. As a result, we observed that the proposed method can suppress $43.7 \%$ vibration in the roll direction and $7.4 \%$ in the pitch direction. The proposed method will reduce the motion sickness caused by the image vibration during teleoperation. In the future, we will realize walking of legged robots on rough terrain by installing CPG feedback.

\section{CONFLICTS OF INTEREST}

The authors declare they have no conflicts of interest.

\section{Authors Introduction}

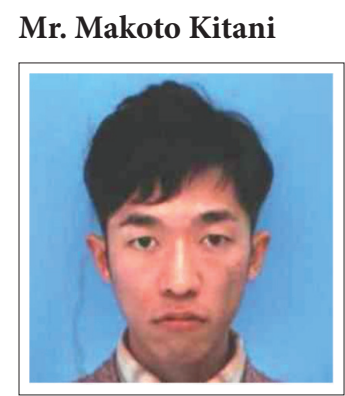

He received Master of Engineering degree from Nagoya Institute of Technology in 2019. His research interests include autonomous control system, system integration and teleoperation system for legged rescue robots.

\section{REFERENCES}

[1] G.S. Stent, W.B. Kristan Jr, W.O. Friesen, C.A. Ort, M. Poon, R.L. Calabrese, Neuronal generation of the leech swimming movement, Science 200 (1978), 1348-1357.

[2] J.T. Buchanan, S. Grillner, Newly identified 'glutamate interneurons' and their role in locomotion in the lamprey spinal cord, Science 236 (1987), 312-314.

[3] H. Kimura, S. Akiyama, K. Sakurama, Dynamic walking on irregular terrain and running on flat terrain of the quadruped using neural oscillator, J. Robot. Soc. Japan 16 (1998), 1138-1145.

[4] Y. Fukuoka, H. Kimura, Realization of dynamic turning motion on irregular terrain of a quadruped robot "TEKKEN4" with neural oscillators, J. Robot. Soc. Japan 72 (2006), 3848-3854.

[5] I. Tsubaki, T. Morita, K. Aizawa, T. Saito, The analysis of oscillatory motion of videos affected by camera shaking for visually induced motion sickness, J. Inst. Electron. Inform. Commun. Eng. 89 (2006), 262-267.

[6] H. Ujike, Visually induced motion sickness, J. Inst. Image. Inform. Television Eng. 61 (2007), 1122-1124.

[7] T. Sun, D. Shao, Z. Dai, P. Manoonpong, Adaptive neural control for self-organized locomotion and obstacle negotiation of quadruped robots, 2018 27th IEEE International Symposium on Robot and Human Interactive Communication (RO-MAN), IEEE, Nanjing, China, 2018, pp. 1081-1086.

[8] Z. Huang, W. Wang, Controller-switching based gait transition for a quadruped robot, 2016 IEEE International Conference on Mechatronics and Automation, IEEE, Harbin, China, 2016, pp. 653-658.

[9] H. Liu, W. Jia, L. Bi, Hopf oscillator based adaptive locomotion control for a bionic quadruped robot, 2017 IEEE International Conference on Mechatronics and Automation, IEEE, Takamatsu, Japan, 2017, pp. 949-954.

[10] P. Sutyasadi, M. Parnichun, Trotting control of a quadruped robot using PID-ILC, IECON 2015 - 41st Annual Conference of the IEEE Industrial Electronics Society, IEEE, Yokohama, Japan, 2015, pp. 4400-4405.

[11] K. Matsushita, Biped Robots on Open Dynamic Engine, 2007, http://www.koj-m.sakura.ne.jp/ode/.

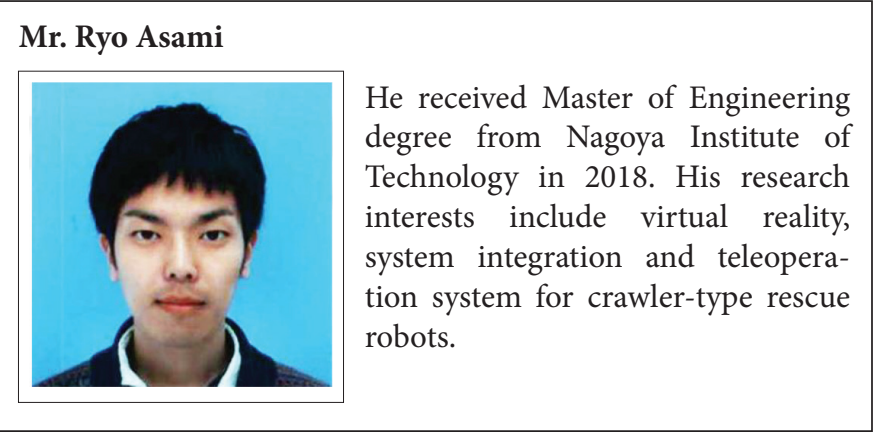




\section{Dr. Noritaka Sato}

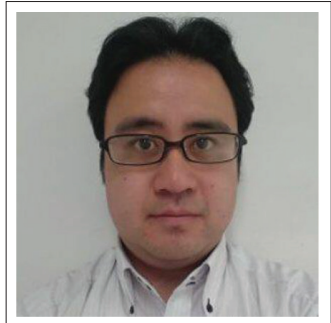

He received Doctor of Engineering degree from The University of Electro-Communications in 2009. He was a Program-Specific Assistant Professor of Kyoto University in 2009-2011, and is an Assistant Professor of Nagoya Institute of Technology since 2011. His research interests include human robot interaction for rescue robots, rehabilitation-assist robots and industrial robots. He is a member of the IEEE, RSJ and SICE.

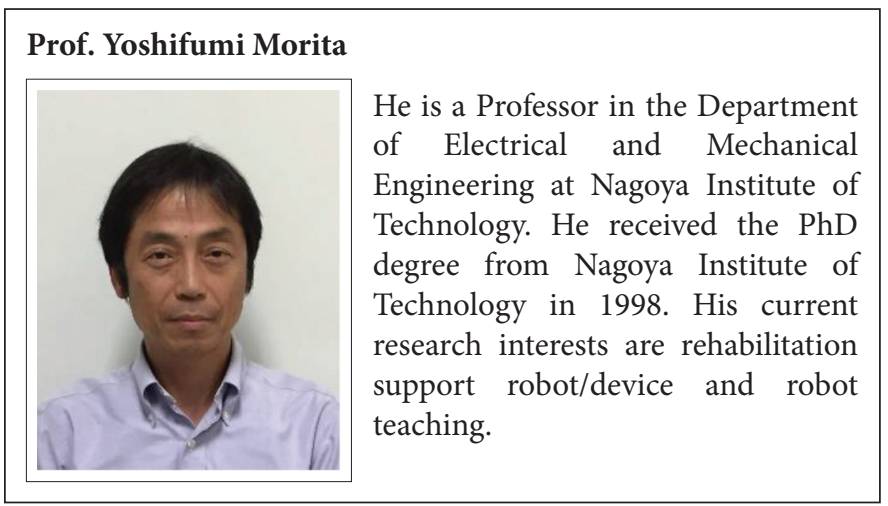

\section{Dr. Tomofumi Fujiwara}

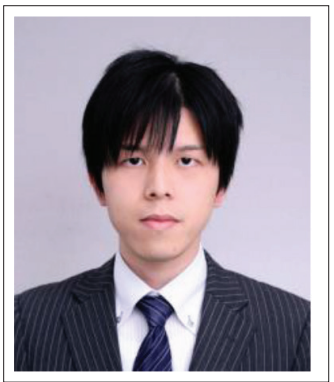

He received his B.S., M.S., and $\mathrm{PhD}$ degrees in Engineering from Okayama University, Japan, in 2010, 2012, and 2016, respectively. From 2014 to 2015 , he was a guest researcher at Technical University of Munich, Germany. From 2016 to 2019, he worked as a ProgramSpecific Assistant Professor (ImPACT) at Kyoto University, Japan. His research interests include rescue robots, interface systems for snake robots and legged robots, real-time 3D laser scanners, and stereoscopic presentation of $3 \mathrm{D}$ point clouds. He is a member of the Robotics Society of Japan (RSJ).

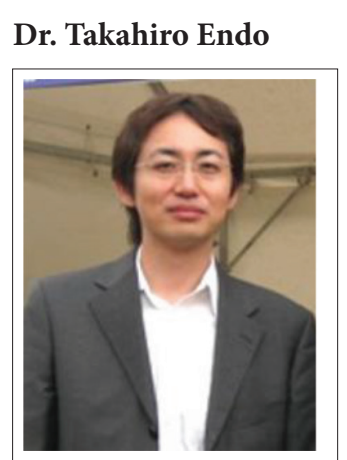

He received the $\mathrm{PhD}$ (Dr. Eng.) degree from the Tokyo Institute of Technology, Japan, in 2006. Since April 2015, he has been with the Department of Mechanical Engineering and Science, Kyoto University, Kyoto, Japan, where he is currently an Associate Professor. His research interests include haptic interfaces, robotics, and the control of infinite dimensional systems.

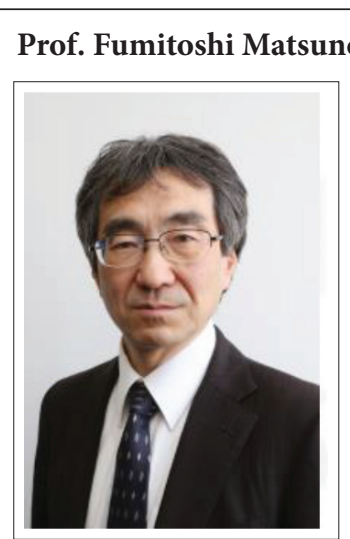

He received the Dr. Eng. from Osaka University in 1986. In 1986, he joined the Department of Control Engineering, Osaka University. Since 2009, he has been a Professor in the Department of Mechanical Engineering and Science, Kyoto University. His current research interests lie in robotics, swarm intelligence, control of distributed parameter system and nonlinear system, and rescue support system in disaster. He is a Fellow member of the SICE, the JSME, the RSJ and a member of the IEEE among other organizations. 